\title{
Looping: solução da lagrangiana, simulação computacional e estratégias didáticas ${ }^{+*}$
}

Nelson Canzian da Silva ${ }^{1}$

Departamento de Física - UFSC

Florianópolis - SC

\section{Resumo}

Este artigo descreve como construir e resolver a lagrangiana para obter as equações de movimento de uma esfera em um looping, tradicional equipamento utilizado em laboratórios de física e outros espaços de divulgação científica para discutir aspectos ligados à conservação da energia. As equações encontradas são codificadas em uma simulação computacional que permite ao usuário escolher alguns parâmetros, tais como altura de lançamento, material e tamanho da esfera. Em resposta, a simulação mostra a sua trajetória e gráficos e tabelas da posição, velocidade, aceleração e energia (em suas diversas manifestações) em função do tempo. São também apresentadas algumas sugestões de uso didático da simulação, e em particular dos gráficos e tabelas produzidos por ela, para buscar ajudar alunos e visitantes a aprofundar sua compreensão das grandezas físicas subjacentes e suas interrelações. A simulação pode ser acessada em $<$ canzian.fsc.ufsc.br/looping >.

Palavras-chave: Simulação; Modelo computacional; Lagrangiana; Looping; Loop-the-loop.

\footnotetext{
Abstract

${ }^{+}$Looping: solution of the lagrangian, computer simulation and didactic strategies

* Recebido: maio de 2015. Aceito: outubro de 2015.

${ }^{1}$ E-mail: nelson.canzian@ufsc.br
}

This paper describes how to build and solve the lagrangian in order to obtain the equations of motion of a sphere in the loop-the-loop track, traditional equipment available in didactic physics laboratories and science communication centers, usually employed to explore the aspects 
of conservation of energy. The equations were coded in a computer simulation through which the user can choose some parameters such as height of launch, material and radius of the sphere. In return, the simulation shows the trajectory of the sphere and plots and tables of its position, velocity, acceleration and energy (in its various manifestations) as a function of the time. Some suggestions of didactic use of the simulation, and specifically the plots and tables it generates, are also presented in order to help students and visitors to better understand the variables and their relationships to each other. The simulation can be viewed at <canzian.fsc.ufsc.br/looping $>$.

Keywords: Simulation; Computer model; Lagrangian; Looping; Loopthe-loop.

\section{Introdução}

O looping (ou loop-the-loop, como é denominado em textos de língua inglesa) é um tradicional equipamento utilizado em laboratórios de física e outros espaços de divulgação científica para discutir aspectos geralmente relacionados à conservação da energia. A Fig. 1 mostra um esquema do equipamento, que é produzido e comercializado por diversas empresas em uma grande variedade de configurações, tamanhos e preços (CIÊNCIA PRIMA, 2014; ARBOR, 2014). Consiste em um trilho no qual rola uma esfera, que desce uma rampa e percorre ou não, dependendo da energia inicial, um círculo vertical (o looping propriamente dito).

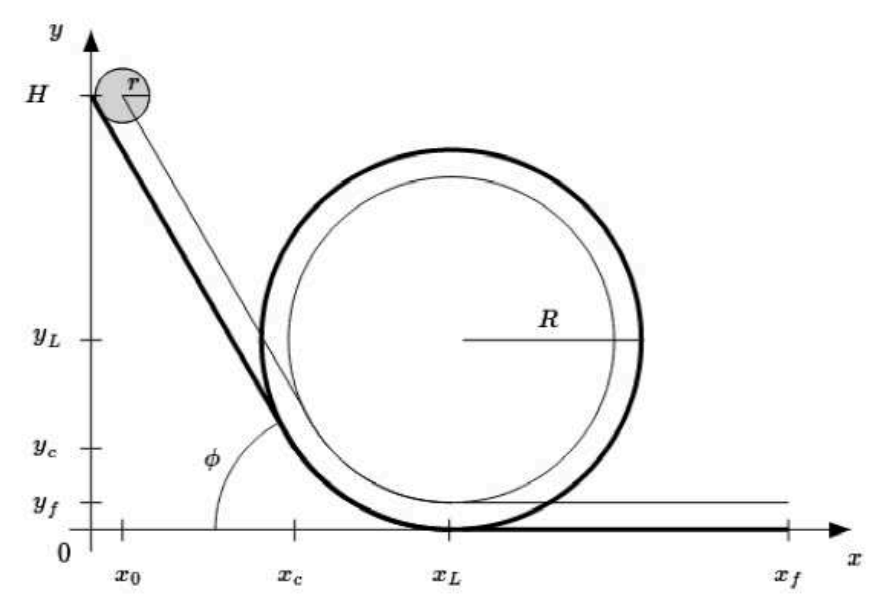

Fig. 1 - Esquema do looping, em que uma esfera de raio rdesce uma rampa inclinada de um ângulo $\phi$ a partir de uma altura $H$, percorrendo ou não o looping de raio $R$, dependendo de sua energia inicial. São identificados também alguns pontos de referência utilizados na simulação: ponto de lançamento $\left(x_{0}, y_{0}=H\right)$, ponto de conexão rampa-looping $\left(x_{c}, y_{c}\right)$, centro do looping $\left(x_{L}, y_{L}\right)$ e ponto final da trajetória $\left(x_{f}, y_{f}\right)$. 
Imagens, vídeos, textos explicativos e estratégias de uso do equipamento são abundantemente encontrados na internet (MATTHIAS, 2013; MIT, 2014; KHAN, 2014, RAGHUNATHAN, 2014). Entretanto, estes textos em geral discutem aspectos relacionados à partição da energia cinética em energia cinética de translação e em energia cinética de rotação e na consequência que isso tem sobre a altura de lançamento necessária para que a esfera percorra o looping completamente. Mais raramente incluem alguns cálculos sobre a dependência da energia com a coordenada angular da esfera no looping.

Em geral, o discurso pode ser resumido como segue: uma esfera de massa $m$ no topo de uma rampa de altura $H$ tem energia potencial gravitacional igual a $E_{p o t}=m g H$. Ao chegar ao final da rampa, esta energia transformou-se em energia cinética de translação $E_{t r n}=(1 / 2) m v^{2}$ e de rotação $E_{r o t}=(1 / 2) I \omega^{2}$, onde $v$ é a velocidade do centro de massa, $I$ o momento de inércia e $\omega=v / r$ a velocidade angular de rotação da esfera, onde $r$ é o seu raio.

Esta relação permite calcular a velocidade do centro de massa no final da rampa, o que possibilita uma discussão sobre os efeitos da energia cinética de rotação. Para o "ponto material" tipicamente encontrado nos livros, a velocidade do centro de massa seria $v=\sqrt{2 g H}$. Levando-se em conta a rotação da esfera, cujo momento de inércia é $I=(2 / 5) m r^{2}$, a velocidade do centro de massa é $v=\sqrt{(5 / 7) 2 g H}$, diferença cerca de $15 \%$ menor com relação ao movimento sem rotação, já razoavelmente perceptível a um observador atento. Esta diferença não depende nem do raio nem da massa da esfera, o que pode surpreender alguns espectadores.

Mas o que dizer sobre os detalhes do movimento da esfera em função do tempo? Como variam a sua velocidade, aceleração e energia ao longo da trajetória? Quanto tempo a esfera leva para chegar ao fim da rampa? A partir desta abordagem da conservação da energia não temos informações sobre a evolução temporal do sistema. Estas informações podem ser obtidas a partir de suas equações de movimento.

Este trabalho tem três principais objetivos: (1) deduzir as equações diferenciais para o movimento da esfera em função do tempo, obtidas a partir do formalismo lagrangiano; (2) apresentar uma simulação de finalidade didática que integra estas equações, mostra o movimento da esfera e gera gráficos e tabelas com dados sobre a posição, velocidade, aceleração e energia e (3) apresentar propostas de utilização da simulação em sala de aula e outros contextos educacionais, buscando estimular o espírito investigativo e aprimorar competências na aquisição, organização, visualização e interpretação de dados.

\section{Materiais e métodos}

\section{Formalismo lagrangiano}

O formalismo lagrangiano provavelmente não é estudado pela maioria dos licenciados em física. Trata-se de um tema normalmente abordado em disciplinas avançadas de mecânica (tipicamente chamadas de "Mecânica Analítica") existentes nos cursos de bacharelado em física. Remetemos os leitores a alguns clássicos livros didáticos de mecânica (GOLDSTEIN, 
1980; SYMON, 1982), ou mesmo uma busca na internet para obter detalhes sobre esse formalismo.

Em linhas gerais, podemos dizer que representa uma alternativa ao formalismo newtoniano, que se fundamente na famosa equação $\vec{F}=d \vec{p} / d t$ (ou $\vec{F}=m \vec{a}$ quando a massa é constante). A escolha do formalismo em geral depende da natureza do problema - às vezes um dos formalismos é muito mais conveniente que o outro - e eventualmente até do gosto de quem se propõe a resolver o problema.

No problema em questão, as equações de movimento podem ser facilmente obtidas para a rampa descendente e para a reta final, onde a força normal é constante, aplicando-se as leis de Newton. No trecho intermediário (o looping propriamente dito) a força normal é variável, e as posições em função do tempo só podem ser calculadas numericamente, o que motivou este trabalho.

A lagrangiana de um sistema é dada pela diferença entre a energia cinética $T$ e a energia potencial $V$ :

$$
L=T-V
$$

Uma vez obtida a lagrangiana é possível chegar às equações diferenciais para o movimento utilizando:

$$
\frac{d}{d t}\left(\frac{\partial L}{\partial \dot{x}_{i}}\right)-\frac{\partial L}{\partial x_{i}}=0
$$

onde $x_{i}$ e $\dot{x}_{i}$ para $i=1, \ldots, N$ são as coordenadas generalizadas utilizadas na descrição do sistema e suas derivadas em relação ao tempo. Esperamos que a análise do problema específico do looping, feita a seguir, deixe um pouco mais claro o que são as coordenadas generalizadas, mas em todo caso remetemos novamente o leitor aos textos didáticos fundamentais para compreender o seu significado com mais profundidade.

O trilho sobre o qual a esfera rola pode ser dividido conceitualmente em quatro partes: (a) uma rampa, do topo da qual é liberada a esfera; (b) um pequeno trecho curvo que faz a ligação entre a rampa e o início do looping; (c) o looping propriamente dito, que é um círculo no plano vertical e que a esfera pode ou não percorrer completamente dependendo de sua energia inicial, e (d) um trecho plano final, no qual, após atravessar o looping a esfera rola sem a ação de forças externas relevantes.

\section{Descendo a rampa}

No caso de uma esfera rolando sem deslizar enquanto desce uma rampa, a energia cinética é dada por:

$$
T=\frac{1}{2} m v^{2}+\frac{1}{2} I \dot{\theta}^{2}
$$

onde $m$ é a massa da esfera, $v$ a velocidade do seu centro de massa, $I$ o momento de inércia e $\dot{\theta}=\omega$ a sua velocidade angular de rotação em torno do centro massa. 
O momento de inércia de uma esfera é dado por $k m r^{2}$, onde $k=2 / 5$ no caso de uma esfera maciça. A velocidade angular e a velocidade do centro de massa estão relacionadas por $\dot{\theta}=v / r$ de modo que a energia cinética pode ser expressa como:

$$
\begin{array}{rlr}
T & = & \frac{1}{2} m v^{2}+\frac{1}{2} k m r^{2} \frac{v^{2}}{r^{2}} \\
T & = & \frac{1}{2}(1+k) m v^{2} \\
T & = & \frac{1}{2}(1+k) m\left(\dot{x}^{2}+\dot{y}^{2}\right) \\
T & =\frac{1}{2}(1+k)\left(1+\tan ^{2} \phi\right) m \dot{x}^{2}
\end{array}
$$

Na equação acima, utilizamos o fato de que o valor da coordenada $y(t)$ do centro de massa da esfera descendo a rampa está relacionado com a coordenada $x(t)$ por

$$
y(t)=H-x(t) \tan \phi
$$

e a velocidade com a derivada disso, ou $\dot{y}=-\dot{x} \tan \phi$ é o ângulo da rampa com a horizontal e $H$ a altura do centro de massa da esfera no ponto de lançamento.

Utilizando esta mesma relação, escrevemos a energia potencial $V$ :

$$
V=m g y=m g(H-x \tan \phi)
$$

E a lagrangiana fica:

$$
L=\frac{1}{2}(1+k)\left(1+\tan ^{2} \phi\right) m \dot{x}^{2}-m g(H-x \tan \phi)
$$

O cálculo das derivadas leva a:

$$
\begin{array}{rlc}
\frac{\partial L}{\partial \dot{x}} & =m\left(1+\tan ^{2} \phi\right)(1+k) \dot{x} \\
\frac{d}{d t} \frac{\partial L}{\partial \dot{x}} & = & m\left(1+\tan ^{2} \phi\right)(1+k) \dot{x} \\
\frac{\partial L}{\partial x} & = & m g \tan \phi
\end{array}
$$

Juntando tudo, as equações diferenciais para $x(t)$ e $y(t)$ ficam:

$$
\begin{aligned}
& \dot{x}=\frac{g}{(1+k)} \frac{\tan \phi}{1+\tan ^{2} \phi}=\frac{g}{(1+k)} \sin \phi \cos \phi \\
& \dot{y}=-\frac{g}{(1+k)} \frac{\tan \phi}{1+\tan ^{2} \phi} \tan \phi=-\frac{g}{(1+k)} \sin ^{2} \phi
\end{aligned}
$$

Como todos os termos à direita são constantes no tempo, as soluções são as esperadas equações para o movimento retilíneo uniformemente variado:

$$
\begin{aligned}
& x(t)=x_{0}+v_{0 x} t+\frac{1}{2} a_{x} t^{2} \\
& y(t)=y_{0}+v_{0 y} t+\frac{1}{2} a_{y} t^{2}
\end{aligned}
$$


Se a coordenada $y$ da posição inicial do centro de massa de uma esfera de raio $x$ está em $y_{0}=H$ em uma rampa que faz um ângulo $\phi$ com a horizontal, a coordenada $x$ da posição inicial do seu centro de massa é dada por $x_{0}=r / \sin \phi$. Tomando $v_{0 x}=0$ e $v_{0 y}=0$, obtemos as equações efetivamente codificadas para este trecho da simulação:

$$
\begin{aligned}
& x(t)=\frac{r}{\sin \phi}+\frac{1}{2} \frac{g}{(1+k)} \sin \phi \cos \phi t^{2} \\
& y(t)=\quad H-\frac{1}{2} \frac{g}{(1+k)} \sin ^{2} \phi t^{2}
\end{aligned}
$$

Estes resultados remetem aos conhecidos resultados para um corpo deslizando sem rolar em um plano inclinado, com algumas pequenas modificações. Primeiro, podemos pensar na rotação, à qual está associada uma energia cinética proporcional à energia cinética de translação $\left(E_{\text {rot }}=k E_{\text {trn }}\right)$, como uma translação em um "planeta" em que a aceleração da gravidade é $g^{\prime}=g /(1+k)$. A seguir, lembramos que nos livros de física este problema usualmente aparece como um problema de MRUV em que as componentes da aceleração são $a_{x^{\prime}}=g^{\prime} \sin \phi$ e $a_{y^{\prime}}=0$ em um sistema de coordenadas $S^{\prime}$ em que o eixo $x^{\prime}$ é paralelo à rampa. No sistema de coordenadas $S$ do laboratório as componentes da aceleração seriam $a_{x}=a_{x^{\prime}} \cos \phi=$ $g^{\prime} \sin \phi \cos \phi$ e $a_{x}=a_{x^{\prime}} \sin \phi=g^{\prime} \sin ^{2} \phi$, iguais às obtidas a partir da lagrangiana.

\section{Percorrendo o looping}

Finda a rampa, a esfera entra no trecho que denominamos conexão rampa-looping. Para equacionar o que acontece neste trecho, entretanto, é conveniente tratar antes o problema do looping propriamente dito, o que fazemos nesta seção, retornando em seguida ao problema do trecho de conexão.

A equação diferencial para o movimento da esfera percorrendo o looping pode ser obtida exatamente como no caso anterior, considerando-se a lagrangiana para o sistema. Neste caso novamente a energia cinética é dada pela soma da energia de translação com a de rotação, mas é mais convenientemente expressa em termos do ângulo $\alpha$ de rotação da esfera no looping, que é o ângulo entre a vertical e a posição da esfera:

$$
\begin{aligned}
T & =\frac{1}{2} m\left(\dot{x}^{2}+\dot{y}^{2}\right)+\frac{1}{2} k m r^{2} \dot{\theta}^{2} \\
T & =\frac{1}{2} m R^{2} \dot{\alpha}^{2}+\frac{1}{2} k m r^{2} \dot{\theta}^{2}
\end{aligned}
$$

Quando a esfera anda uma distância $R \alpha$ no looping, um ponto em sua superfície anda uma distância $r \theta$, de modo que $\dot{\theta}=(R / r) \dot{\alpha}$, o que permite escrever a expressão para a energia cinética apenas em termos de $\alpha$ :

$$
T=\frac{1}{2} m R^{2} \dot{\alpha}^{2}+\frac{1}{2} k m r^{2} \frac{R^{2}}{r^{2}} \dot{\alpha}^{2}=\frac{1}{2} m(1+k) R^{2} \dot{\alpha}^{2}
$$

A energia potencial é dada por: 


$$
V=m g(R-R \cos \alpha)
$$

A lagrangiana dada por:

$$
L=T-V=\frac{1}{2} m(1+k) R^{2} \dot{\alpha}^{2}-m g(R-R \cos \alpha)
$$

As derivadas requeridas são:

$$
\begin{aligned}
\frac{\partial L}{\partial \dot{\alpha}} & =m(1+k) R^{2} \dot{\alpha} \\
\frac{d}{d t} \frac{\partial L}{\partial \dot{\alpha}} & =m(1+k) R^{2} \dot{\alpha} \\
\frac{\partial L}{\partial \alpha} & =-m g R \sin \alpha
\end{aligned}
$$

Juntando tudo, obtemos:

$$
\ddot{\alpha}=-\frac{g}{(1+k) R} \sin \alpha
$$

Esta é a equação diferencial para o pêndulo simples em um mundo com gravidade $g^{\prime}=g /(1+k)$, no ponto onde usualmente é feita a aproximação de pequenos ângulos $\sin \alpha \approx \alpha$. No nosso caso, como queremos que a esfera percorra todos os ângulos, obviamente não podemos fazer esta aproximação. Sem a aproximação, a equação diferencial não tem solução analítica e empregaremos métodos numéricos para integrá-la. Neste trabalho, utilizamos o tradicional método de Runge-Kutta de $4^{\mathrm{a}}$ ordem partindo de $\alpha=0$ e prosseguindo a integração até que a esfera complete o círculo atingindo $\alpha=2 \pi$, caso tenha energia inicial suficiente para tanto, ou descole do looping quando a aceleração centrípeta $v^{2} / R$ igualar-se à gravidade $g$.

\section{Conexão rampa-looping}

Num sistema físico realista a passagem da rampa para o looping não se dá de forma abrupta. Na nossa modelagem a rampa também não termina de forma abrupta em $y=0$, quando $x=H / \tan \phi$, mas em um arco de círculo. Por conveniência, vamos supor que o raio deste arco de círculo seja igual ao raio do looping. O problema consiste em encontrar as coordenadas $\left(x_{c}\right.$, $y_{c}$ ) do ponto em que a tangente ao arco é exatamente igual ao ângulo de inclinação da reta. Considerando que a reta que liga o início da rampa $(0, H)$ a $\left(x_{c}, y_{c}\right)$ é perpendicular à reta que liga $\left(x_{c}, y_{c}\right)$ ao centro do círculo $\left(x_{L}, y_{L}\right)$, um pouco de trigonometria permite calcular as coordenadas $\left(x_{c}, y_{c}\right)$ do ponto de conexão:

$$
\begin{aligned}
& x_{c}=\frac{H-R(1-\cos \phi)}{\tan \phi} \\
& y_{c}=R(1-\cos \phi)
\end{aligned}
$$

Desse modo, quando o centro de massa da esfera atinge essas coordenadas ao descer a rampa, o cálculo da trajetória passa a ser feito pela metodologia discutida na seção anterior (Percorrendo o loooping). 


\section{Reta final}

Uma vez tendo percorrido o looping a esfera entra na reta final, onde seu centro de massa realiza um trivial movimento retilíneo uniforme com equações de movimento dadas por:

$$
\begin{array}{llc}
x(t) & = & x_{L}+v_{x L} t \\
y(t) & = & r
\end{array}
$$

sendo $v_{x L}=\sqrt{2 g H /(1+k)}$, no caso de um sistema sem atrito.

\section{Oscilação ou queda livre}

Caso a esfera não tenha energia suficiente para percorrer o looping, são dois os seus possíveis destinos: oscilar no looping ou cair em queda livre. Caso a energia inicial não seja suficiente para que passe de $\alpha=\pi / 2$ a esfera irá oscilar seguindo as equações de movimento encontradas para o movimento no looping, que serão integradas numericamente. Caso ultrapasse este ângulo mas não tenha energia suficiente para atravessar o looping, vai descolar do trilho e cair em queda livre, girando. Neste caso, as equações de movimento são as do lançamento oblíquo:

$$
\begin{array}{llc}
x(t) & = & x_{d}+v_{x d} t \\
y(t) & = & y_{d}+v_{y d} t+\frac{1}{2} g t^{2}
\end{array}
$$

nas quais $x_{d}=R \sin \alpha_{d}$ e $y_{d}=R \cos \alpha_{d}$ são as coordenadas do ponto em que ocorre o deslocamento e $v_{x d}=R \dot{\alpha} \cos \alpha$ e $v_{y d}=R \dot{\alpha} \sin \alpha$ as componentes da velocidade do centro de massa neste ponto.

Mas qual a condição para que a esfera descole do trilho, que indica quando o modelo utilizado no cálculo deve ser substituído?

Quando a esfera está a um ângulo $\alpha>\pi / 2$, o equilíbrio de forças pode ser escrito em termos da componente radial e da componente tangencial. Para a componente radial temos a normal $N$ e a parte do peso dada por $-m g \cos \alpha$ cuja soma deve ser igual à força centrípeta. Para a componente tangencial há somente a parte do peso dada por $m g \sin \alpha$, que é igual à massa da esfera multiplicada pela aceleração que modifica o módulo de sua velocidade tangencial à medida que sobe o looping:

$$
\begin{aligned}
N-m g \cos \alpha & =\frac{m v^{2}}{R} \\
m g \sin \alpha & =m a_{t}
\end{aligned}
$$

O descolamento ocorre quando a normal $N$ se anula e, portanto:

$$
-g \cos \alpha=\frac{v^{2}}{R}
$$


É possível encontrar o valor do ângulo para o qual isto acontece em função da altura de lançamento e do raio do looping aplicando a conservação da energia. No ponto em que há o descolamento o balanço da energia e a equação acima levam a:

$$
\begin{array}{rlrl}
m g H & =m g(R-R \cos \alpha)+\frac{1}{2} m(1+k) v^{2} \\
v^{2} & = & \frac{2 g}{(1+k)}[H-R(1-\cos \alpha)] \\
-R g \cos \alpha & = & \frac{2 g}{(1+k)}[H-R(1-\cos \alpha)] \\
\cos \alpha & = & -\frac{2}{(3+k)}\left(\frac{H}{R}-1\right)
\end{array}
$$

A altura de lançamento é obviamente o parâmetro relevante, pois define a energia total $E=m g H$ inicial no sistema. Sua relação com o raio do looping determina se a esfera percorrerá ou não o looping. A altura mínima $H_{\text {min }}$ de lançamento para que isto aconteça num sistema sem atrito pode ser calculada utilizando a conservação da energia mecânica e considerando que a esfera fará o looping se a sua velocidade no topo provê-la de aceleração centrípeta no mínimo igual à aceleração da gravidade:

$$
\begin{array}{rlc}
m g H_{\text {min }} & = & m g(2 R)+\frac{1}{2}(1+k) m v^{2} \\
\frac{v^{2}}{R} & = & g \\
m g H & = & m g(2 R)+\frac{1}{2}(1+k) m R g \\
H_{\text {min }} & = & 2 R+\frac{1}{2}(1+k) R
\end{array}
$$

O ângulo de inclinação é totalmente irrelevante quando não consideramos perdas de energia ao longo da trajetória. Quando estas são consideradas, ângulos menores levam a percursos maiores na rampa e, portanto, a uma maior dissipação da energia.

\section{Implementação computacional}

A implementação computacional foi feita utilizando HTML (HICKSON et al., 2014), CSS (BOS et al., 2011) e JavaScript (WIRFS-BROCK et al., 2015), que permite uma integração natural entre texto, ilustrações dinâmicas, gráficos e elementos de interatividade (botões, barras deslizantes etc.). Para a apresentação dos gráficos foi utilizado o pacote FLOT (LAURSEN; SCHUNUR, 2014).

No programa, o problema está dividido em rotinas que implementam as equações da seção anterior. A primeira rotina, que controla a descida da rampa, utiliza as equações para o 
movimento retilíneo uniformemente acelerado levando em conta a rotação da esfera. A segunda rotina implementa o algoritmo numérico de Runge-Kutta de 4⿳亠丷a ordem (PRESS et al., 2007) para integrar a equação diferencial e propagar a trajetória na conexão rampa-looping. A terceira rotina utiliza o mesmo método numérico para propagar a trajetória no looping, avaliando o que acontece nele (retorno sem queda livre, queda livre ou travessia completa) e encaminhando o processamento para rotinas que implementam as equações para cada caso.

Durante todo o cálculo os valores das sucessivas posições $\left(x_{c m}\right.$ e $\left.y_{c m}\right)$, velocidades $\left(v_{x c m}\right.$ e $\left.v_{y c m}\right)$, acelerações $\left(a_{x c m}\right.$ e $\left.a_{y c m}\right)$ e a divisão da energia nas suas diversas manifestações $\left(E_{\text {pot }}, E_{\text {cin }}, E_{\text {tot }}, E_{\text {tra }}\right.$ e $\left.E_{\text {rot }}\right)$ são armazenados em intervalos de 1 milissegundo (0,001 s). Os pontos nos gráficos, no entanto, são apresentados a intervalos de 1 décimo de segundo $(0,1 \mathrm{~s})$ ou um centésimo de segundo $(0,01 \mathrm{~s})$, de acordo com a escolha do usuário. Nos trechos em que o movimento é circular (conexão rampa-looping e looping) o algoritmo de Runge-Kutta utiliza um passo de 10 microssegundos $(0,00001 \mathrm{~s})$, suficiente para evitar variações na energia total no intervalo considerado.

\section{Resultados}

\section{Interface com o usuário}

A Fig. 2 mostra a interface com o usuário do programa de simulação. Nela, o usuário pode escolher: (a) o material e (b) o raio da esfera, combinação que permite explorar a dependência dos resultados com a massa da esfera; (c) a taxa de amostragem do movimento e (d) a altura de lançamento, dada em função do raio do looping.

\section{Trajetórias}

A Fig. 3 mostra desenhos equivalentes aos resultados que seriam obtidos com técnicas fotográficas utilizando uma fonte de luz estroboscópica para registrar o movimento da esfera ao longo do looping. No quadro superior está representada uma trajetória à qual nos referiremos como de "altura baixa" de lançamento, em que a esfera não tem energia suficiente para subir o looping ao ponto de descolar-se dele ou de percorrê-lo completamente. Nesse caso, na ausência de forças dissipativas, a esfera oscilaria entre o ponto de lançamento e o ponto de mesma altura no looping. No quadro central está representada uma trajetória à qual nos referiremos como de "altura intermediária", em que a esfera tem energia suficiente para passar do primeiro quadrante do looping mas não para percorrê-lo completamente. Nesse caso, a esfera "descola" do looping e cai em um movimento semelhante ao denominado por lançamento horizontal nos textos de física. No quadro inferior está representada uma trajetória à qual nos referiremos como de "altura elevada" de lançamento, em que a esfera tem energia suficiente para percorrer o looping completamente. 


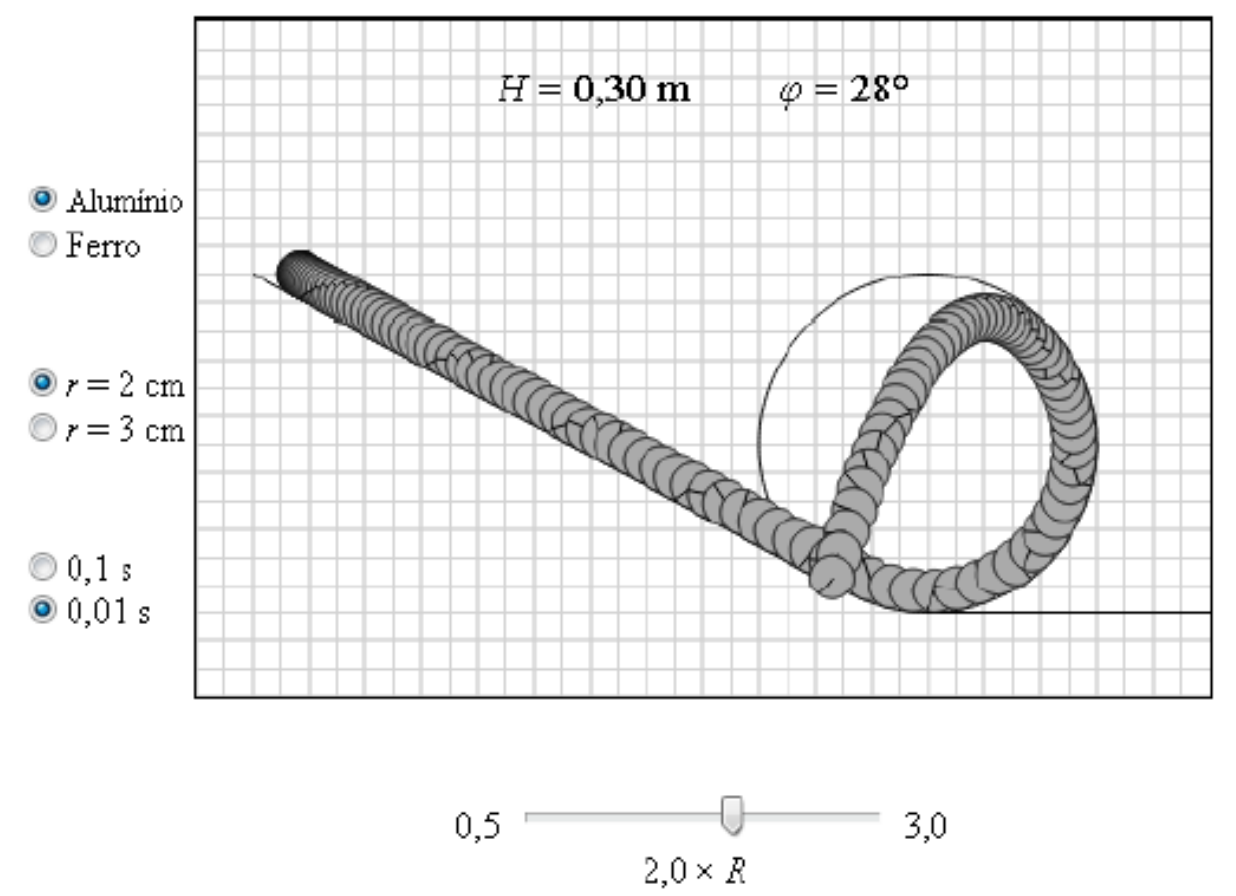

Fig. 2 - Interface do programa de simulação. O usuário pode escolher diferentes densidades de materiais (alumínio ou ferro), diferentes tamanhos da esfera $(r=2 \mathrm{~cm}$ ou $r=3$ $\mathrm{cm})$, diferentes intervalos de amostragem dos resultados $(0,1 \mathrm{~s}$ ou $0,01 \mathrm{~s})$ e diferentes alturas de lançamento (o que define o ângulo de lançamento, uma vez que o raio $R$ do looping é fixo).

A Fig. 3 mostra apenas o que o espectador pode perceber com seus próprios olhos explorando o equipamento. A intenção desse trabalho, entretanto, é oferecer subsídios e estratégias para permitir a ampliação da percepção das características do movimento através da análise dos gráficos das componentes dos vetores posição, velocidade e aceleração e das várias manifestações da energia (cinética, potencial, translacional, rotacional e total) do sistema.

\section{Posição em função do tempo}

Apesar da dificuldade que muitos estudantes têm para interpretar gráficos, o gráfico da posição em função do tempo é provavelmente o de mais fácil interpretação. A percepção da variação da posição de um objeto é direta, e, exceto em situações muito peculiares, não exige abstrações complexas. Entretanto, representar separadamente as coordenadas e o tempo 


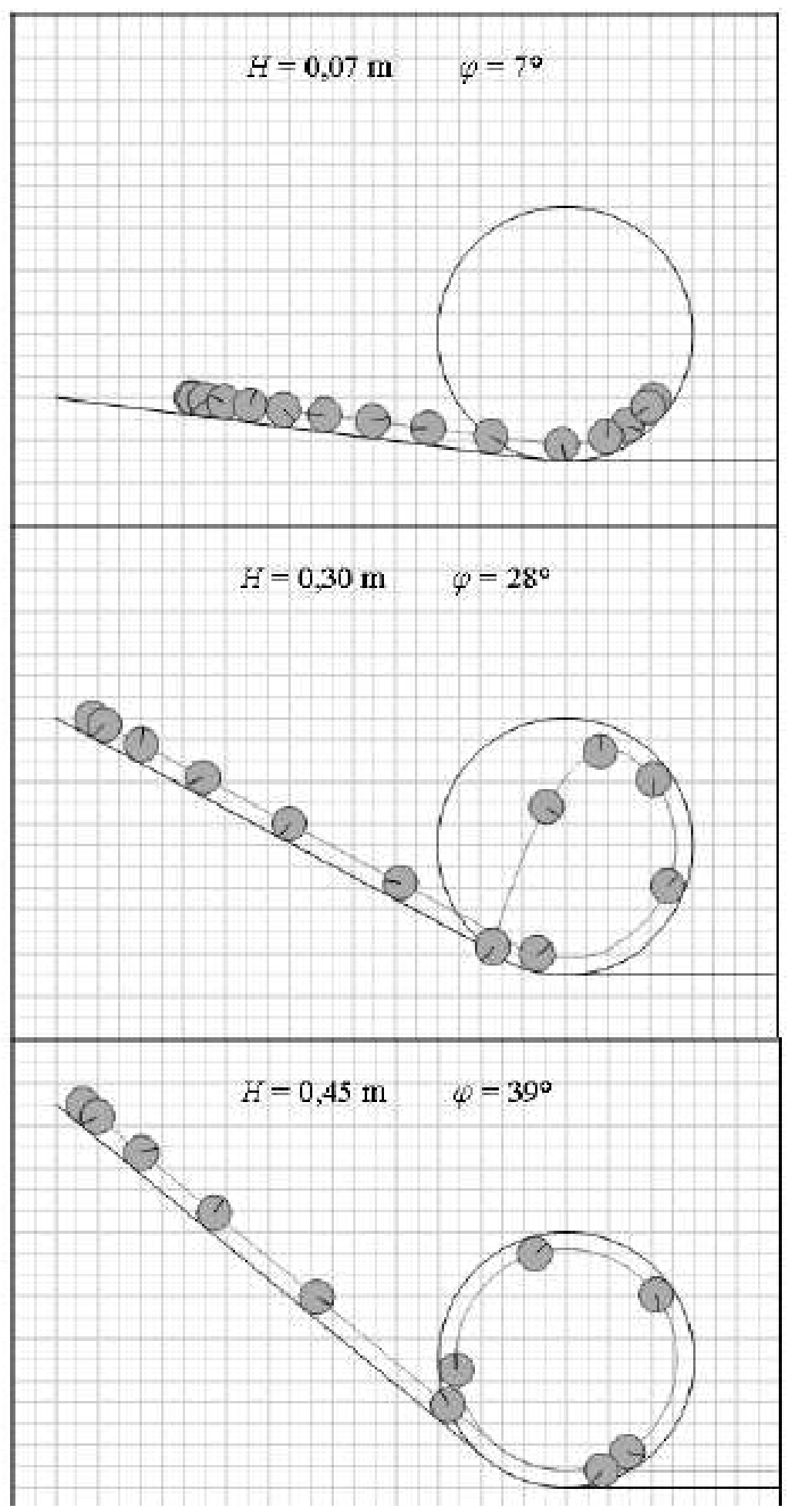

Fig. 3 - As imagens geradas pelo programa simulam o resultados que seriam obtidos com técnicas fotográficas utilizando uma fonte de luz estroboscópica para registrar o movimento da esfera ao longo do looping para três situações que denominaremos altura baixa de lançamento (quadro superior), altura intermediária de lançamento (quadro central) e altura elevada de lançamento (quadro inferior). 
em um plano cartesiano consiste em uma abstração relativamente sofisticada, mas é justamente para a construção dessa competência que esperamos contribuir.

Ao ajudar os alunos a interpretar o que veem no gráfico, o mediador pode, com razoável tranquilidade, utilizar termos como "subir" e "descer" e "esquerda" e "direita", uma vez que há uma correspondência entre os seus significados e o que acontece com a esfera e com as linhas do gráfico. Vejamos o que acontece para o caso de um lançamento a baixa altura (Fig. 4, quadro superior). A curva correspondente à coordenada $x$ (marcada com círculos) "sobe" à medida que o tempo passa - é a esfera indo sempre para a direita - até certo ponto em que parece nem subir nem descer (um "patamar"), que corresponde ao instante em que a esfera para no looping e depois começa a "descer", voltando em direção à rampa. Para a interpretação da curva correspondente à coordenada $y$ (marcada com quadrados), a correspondência é ainda mais direta: tal como a linha, a esfera desce, sobe e desce novamente.

As curvas para a coordenada $y$ têm a mesma estrutura nos três casos (descida seguida de um "morro"). Entretanto, exceto no primeiro trecho, representam desfechos diferentes. A diferença fica evidente quando comparamos as curvas para a coordenada $x$, especialmente os trechos em que varia linearmente, decrescendo para o lançamento a altura intermediária (a queda livre com alguma velocidade à esquerda) e crescendo para o lançamento a altura elevada (o movimento retilíneo uniforme do centro de massa no trecho horizontal final do equipamento).

No primeiro trecho (a rampa), apesar de o movimento acontecer em linha reta (inclinada), as linhas nos gráficos são curvas suaves (a variação da posição é quadrática com o tempo $\left(\propto g t^{2}\right)$. É interessante comparar esse comportamento com o que acontece no último trecho no caso de lançamentos altos $\left(H>2,7 R_{L}\right)$. Nesse caso, a esfera também percorre uma reta (horizontal) mas, diferentemente do primeiro trecho, os gráficos mostram retas (inclinada no caso de $x)$.

\section{Velocidade em função do tempo}

O módulo da velocidade ( $|v|_{c m}$, marcado por triângulos na Fig. 5) é bem intuitivo: a velocidade cresce enquanto a esfera desce a rampa, diminui quando começa a subir o looping e atinge o máximo no final do movimento.

Em todos os casos há um mínimo. No lançamento a baixa altura, o mínimo está associado ao ponto em que a esfera para no looping e a seguir retorna em direção à rampa (as componentes $v_{x \mathrm{~cm}}$ e $v_{y c m}$ se anulam neste ponto). No lançamento a altura intermediária, o mínimo está associado a um momento um pouco posterior ao momento em que a esfera "descola" do looping $\left(v_{y c m}=0\right)$. No lançamento a altura elevada o mínimo se dá quando a esfera está no alto do looping ( $v_{y c m}$ é nula e $v_{x c m}$ é, numericamente, máxima). 

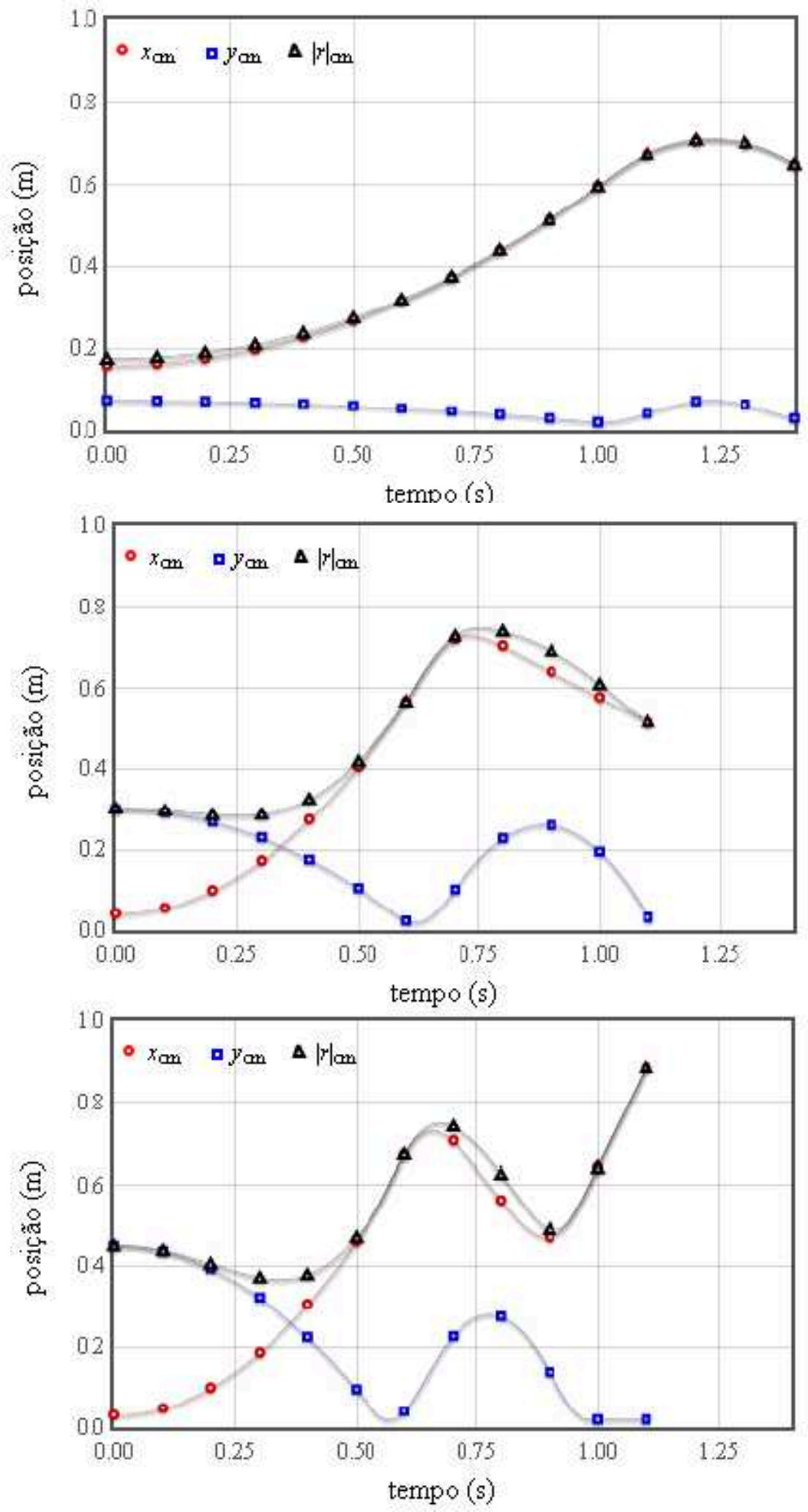

Fig. 4 - Posições x e y do centro de massa da esfera em função do tempo para lançamentos a altura baixa (quadro superior), intermediária (quadro central) e elevada (quadro inferior). 

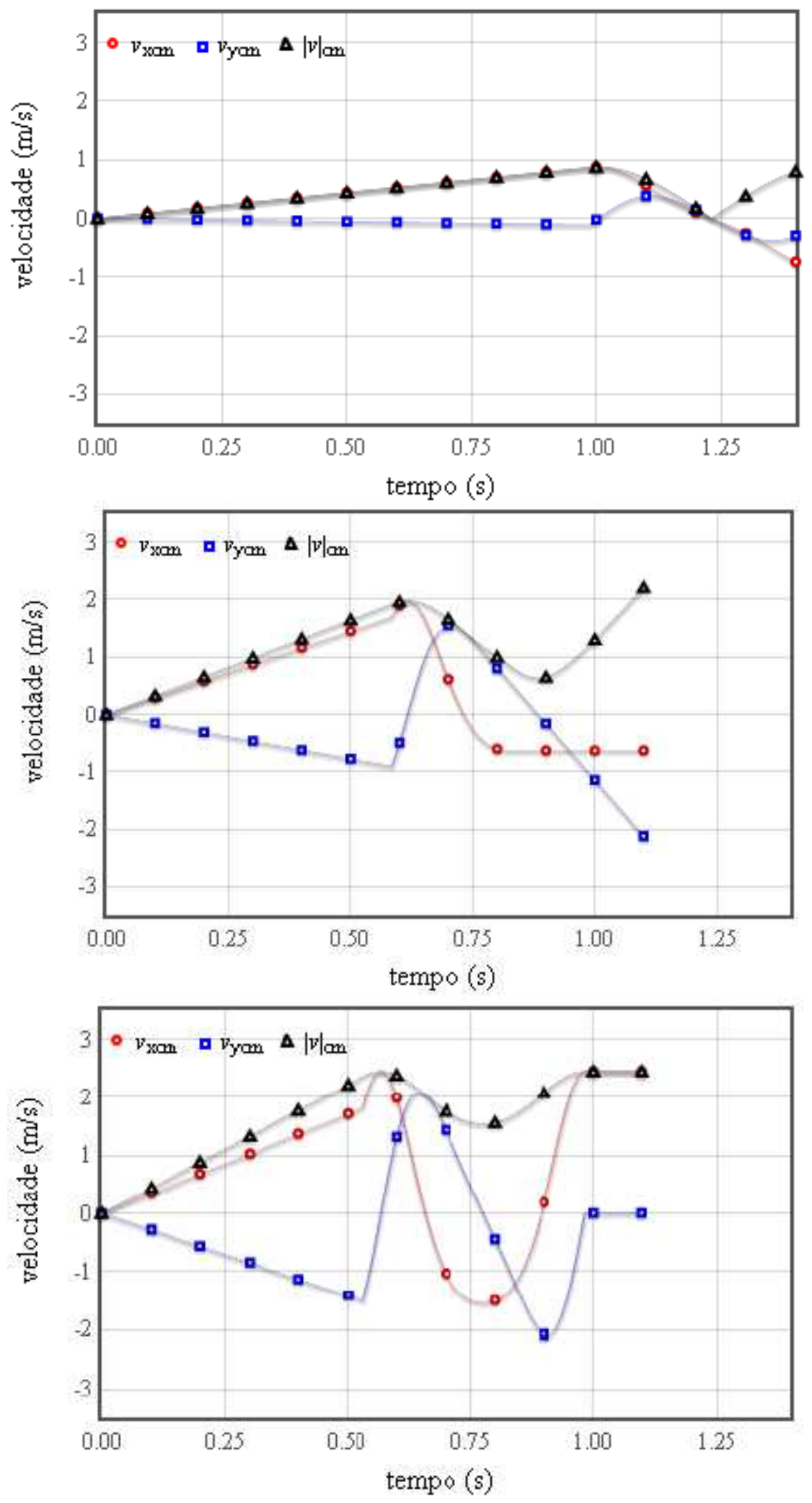

Fig. 5-Módulo $|v|_{c m}$, e componentes $v_{x c m}$, e $v_{y c m}$ da velocidade do centro de massa da esfera em função do tempo para lançamentos a altura baixa (quadro superior), intermediária (quadro central) e elevada (quadro inferior). 
As componentes $v_{x c m}$ e $v_{y c m}$ têm variações abruptas nos pontos de conexão rampalooping e looping-reta-final. Para a componente $v_{y c m}$ nos gráficos para lançamento a altura intermediária e altura elevada, antes da conexão rampa-looping, a velocidade vertical cresce (negativamente) linearmente, mas deve passar rapidamente a zero quando atinge o fundo do looping. No caso do lançamento a altura elevada, o mesmo se dá na transição looping-reta-final. Apesar de pouco usual se comparado ao que normalmente se encontra nos livros de física, essas variações abruptas estão associadas a mudanças bruscas na aceleração e, consequentemente, na força que a estrutura do looping tem que fazer sobre a esfera (pense no que aconteceria nesses pontos em uma pista de borracha).

\section{Aceleração em função do tempo}

Os gráficos para o módulo $|a|_{c m}$ e as componentes $a_{x c m}$ e $a_{y c m}$ para as três situações de lançamento são mostrados na Fig. 6.

No lançamento para altura baixa, $a_{x c m}$ é pequena, e a componente $a_{y c m}$ domina. Percebe-se que a componente $a_{x c m}$ não é nula pelo pequeno deslocamento para cima que se vê perto dos 0,80 segundos, quando a esfera atinge o fundo do looping e a aceleração se anula. Depois desse ponto, a componente $a_{x c m}$ oscila um pouco enquanto a esfera sobe o pequeno trecho do looping e retorna ao fundo, quando termina a simulação. A componente $a_{y c m}$ fica cada vez mais negativa à medida que a esfera sobe o looping, atingindo seu máximo no ponto de retorno (em que a tangente à trajetória está mais próxima possível da vertical), voltando a zero quando chega novamente ao fundo do looping.

Para o lançamento a uma altura intermediária, o gráfico é essencialmente o mesmo até cerca de aproximadamente $0,8 \mathrm{~s}$, exceto por uma amplificação da escala vertical (a aceleração é maior) e uma contração da escala horizontal (a esfera é mais rápida). No entanto, nesse instante a esfera descola-se do trilho e passa a fazer o movimento de um projétil, sujeita somente à aceleração da gravidade.

No lançamento a uma altura elevada, o gráfico é essencialmente o mesmo até cerca de 0,7 s, com uma maior amplificação da escala vertical e contração da escala horizontal. Como nessa situação ela tem energia suficiente para percorrer o looping, nota-se a diferença de fase das componentes $a_{x c m}$ e $a_{y c m}$ : ambas se anulam em $\alpha=0, \pi$ e $2 \pi$ e uma é máxima e a outra é nula nos em $\alpha=\pi / 2$ e $3 \pi / 2$. Na reta final, o movimento do centro de massa é retilíneo e uniforme, com aceleração nula.

\section{Energia em função do tempo}

Os gráficos para as várias manifestações da energia (Fig. 7) parecem muito similares para as três situações de lançamento. As características mais marcantes, em todos os casos, são: 

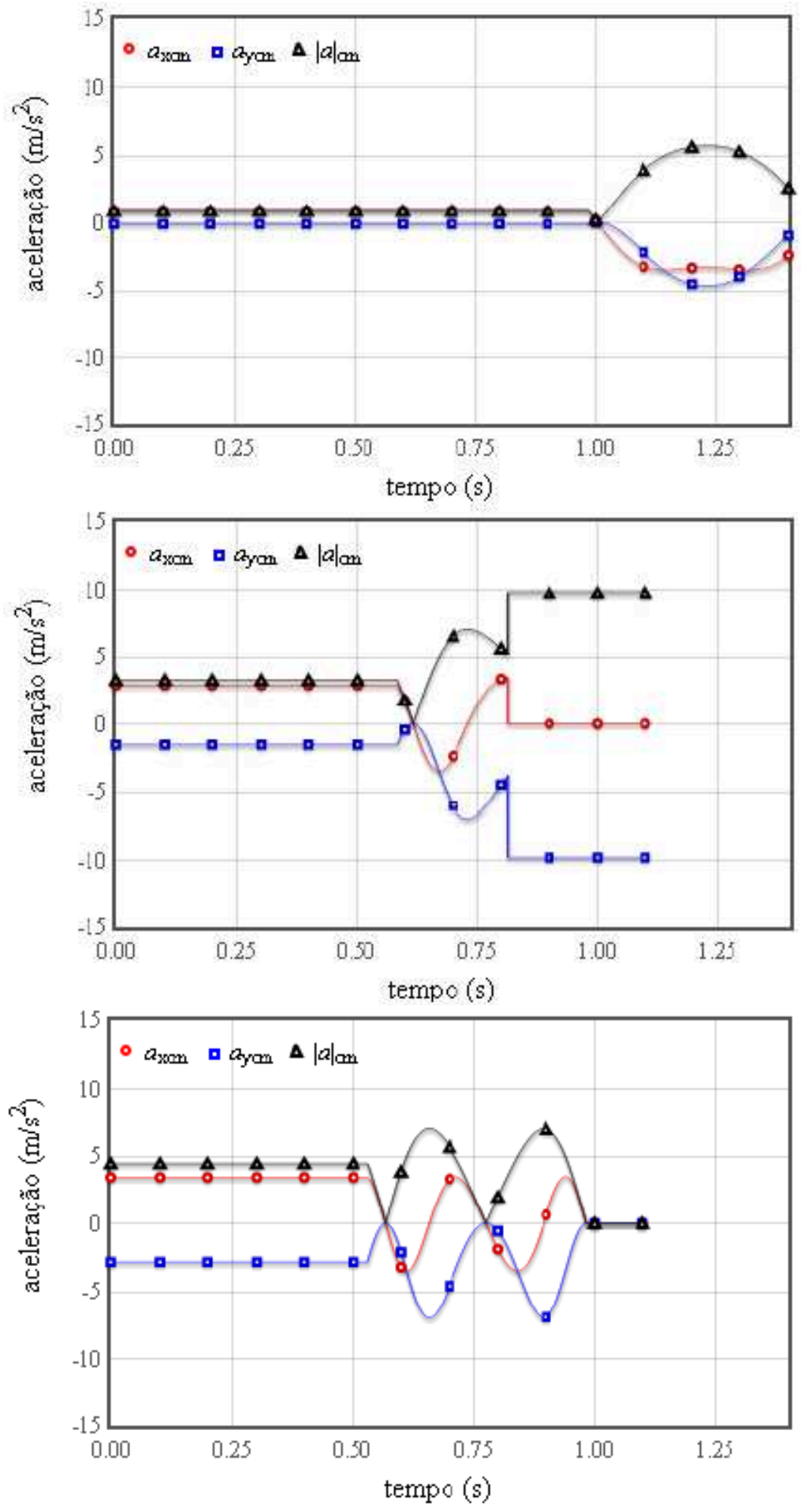

Fig. 6 - Módulo $|a|_{c m}$ e componentes $a_{x c m}$ e $a_{y c m}$ da aceleração do centro de massa da esfera em função do tempo para lançamentos a altura baixa (quadro superior), intermediária (quadro central) e elevada (quadro inferior). 

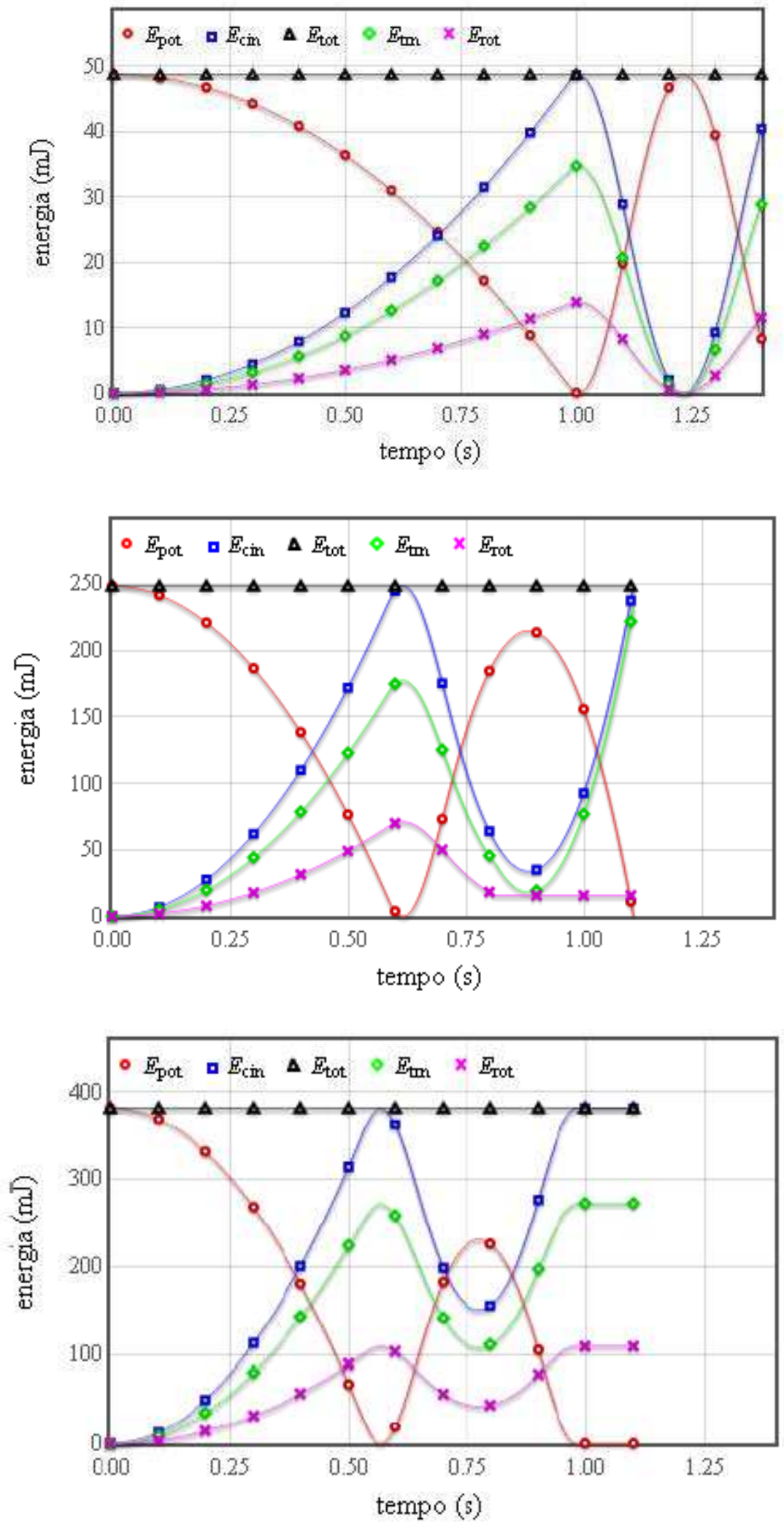

Fig. 7 - Energia potencial, cinética, total, de translação e de rotação da esfera em função do tempo para lançamentos a altura baixa (quadro superior), intermediária (quadro central) e elevada (quadro inferior). 
(a) a constância da energia total (como era de se esperar);

(b) o fator de escala entre a energia cinética total e as energias cinéticas de translação e rotação $\left(E_{\text {rot }}=(2 / 7) E_{\text {cin }}, E_{t r n}=(5 / 7) E_{\text {cin }}\right)$;

(c) a complementaridade entre a energia cinética total e a energia potencial, cuja soma sempre resulta na energia total.

\section{Tabelas de dados}

O programa também gera tabelas com os valores das variáveis cinemáticas (posição, velocidade e aceleração) do movimento e com os valores da distribuição da energia entre as suas classificações (potencial, cinética, total, cinética de rotação e cinética de rotação). O programa permite imprimir estas informações em passos de $0,1 \mathrm{~s}$ ou de $0,01 \mathrm{~s}$, a critério do usuário. As tabelas 1 e 2 mostram os resultados para o caso de um lançamento de uma altura intermediária (em que a esfera "descola" do looping e cai em queda livre) em intervalos de $0,1 \mathrm{~s}$.

Tabela 1 - Valores das variáveis cinemáticas (componentes e módulo da posição, velocidade e aceleração) da esfera ao longo de uma trajetória iniciada de uma altura intermediária (em que a esfera "descola" do looping e cai em queda livre).

\begin{tabular}{|c|c|c|c|c|c|c|c|c|}
\hline $\begin{array}{c}\boldsymbol{t} \\
(\mathbf{s})\end{array}$ & $\begin{array}{c}\boldsymbol{x}_{\mathbf{c m}} \\
(\mathbf{m})\end{array}$ & $\begin{array}{c}\boldsymbol{y}_{\mathbf{c m}} \\
(\mathbf{m})\end{array}$ & $\begin{array}{c}\boldsymbol{v}_{\mathbf{x c m}} \\
(\mathbf{m} / \mathbf{s})\end{array}$ & $\begin{array}{c}\boldsymbol{v}_{\mathbf{c c m}} \\
(\mathbf{m} / \mathbf{s})\end{array}$ & $\begin{array}{c}\boldsymbol{v}_{\mathbf{c m}} \\
(\mathbf{m} / \mathbf{s})\end{array}$ & $\begin{array}{c}\boldsymbol{a}_{\mathbf{x c m}} \\
\left(\mathbf{m} / \mathbf{s}^{\mathbf{2}}\right)\end{array}$ & $\begin{array}{c}\boldsymbol{a}_{\mathbf{y c m}} \\
\left(\mathbf{m} / \mathbf{s}^{\mathbf{2}}\right)\end{array}$ & $\begin{array}{c}\boldsymbol{a}_{\mathbf{c m}} \\
\left(\mathbf{m} / \mathbf{s}^{\mathbf{2}}\right)\end{array}$ \\
\hline 0.00 & 0.043 & 0.300 & 0.000 & 0.000 & 0.000 & 2.91 & -1.55 & 3.29 \\
\hline 0.10 & 0.057 & 0.292 & 0.291 & -0.155 & 0.329 & 2.91 & -1.55 & 3.29 \\
\hline 0.20 & 0.101 & 0.269 & 0.581 & -0.310 & 0.659 & 2.91 & -1.55 & 3.29 \\
\hline 0.30 & 0.173 & 0.230 & 0.872 & -0.465 & 0.988 & 2.91 & -1.55 & 3.29 \\
\hline 0.40 & 0.275 & 0.176 & 1.163 & -0.620 & 1.318 & 2.91 & -1.55 & 3.29 \\
\hline 0.50 & 0.406 & 0.106 & 1.453 & -0.775 & 1.647 & 2.91 & -1.55 & 3.29 \\
\hline 0.60 & 0.567 & 0.024 & 1.901 & -0.498 & 1.965 & 1.72 & -0.45 & 1.77 \\
\hline 0.70 & 0.721 & 0.102 & 0.610 & 1.548 & 1.664 & -2.39 & -6.06 & 6.51 \\
\hline 0.80 & 0.704 & 0.228 & -0.602 & 0.803 & 1.004 & 3.36 & -4.48 & 5.60 \\
\hline 0.90 & 0.641 & 0.261 & -0.631 & -0.161 & 0.651 & 0.00 & -9.80 & 9.80 \\
\hline 1.00 & 0.578 & 0.196 & -0.631 & -1.141 & 1.304 & 0.00 & -9.80 & 9.80 \\
\hline 1.10 & 0.515 & 0.032 & -0.631 & -2.121 & 2.213 & 0.00 & -9.80 & 9.80 \\
\hline
\end{tabular}


Tabela 2 - Valores associados às várias manifestações da energia (potencial, cinética, total, cinética de translação e cinética de rotação) da esfera ao longo de uma trajetória iniciada de uma altura intermediária (em que a esfera "descola" do looping e cai em queda livre).

\begin{tabular}{|c|c|c|c|c|c|}
\hline $\begin{array}{c}\boldsymbol{t} \\
(\mathbf{s})\end{array}$ & $\begin{array}{c}\boldsymbol{E}_{\text {pot }} \\
(\mathbf{m J})\end{array}$ & $\begin{array}{c}\boldsymbol{E}_{\text {cin }} \\
(\mathbf{m J})\end{array}$ & $\begin{array}{c}\boldsymbol{E}_{\text {tot }} \\
(\mathbf{m J})\end{array}$ & $\begin{array}{c}\boldsymbol{E}_{\text {trn }} \\
(\mathbf{m J})\end{array}$ & $\begin{array}{c}\boldsymbol{E}_{\text {rot }} \\
(\mathbf{m J})\end{array}$ \\
\hline 0.00 & 248.0 & 0.0 & 248.0 & 0.0 & 0.0 \\
\hline 0.10 & 241.0 & 6.9 & 248.0 & 4.9 & 2.0 \\
\hline 0.20 & 221.0 & 27.5 & 248.0 & 19.6 & 7.8 \\
\hline 0.30 & 186.0 & 61.9 & 248.0 & 44.2 & 17.7 \\
\hline 0.40 & 138.0 & 110.0 & 248.0 & 78.5 & 31.4 \\
\hline 0.50 & 76.5 & 172.0 & 248.0 & 123.0 & 49.1 \\
\hline 0.60 & 3.8 & 245.0 & 248.0 & 175.0 & 69.9 \\
\hline 0.70 & 73.0 & 175.0 & 248.0 & 125.0 & 50.1 \\
\hline 0.80 & 184.0 & 63.8 & 248.0 & 45.6 & 18.2 \\
\hline 0.90 & 213.0 & 34.9 & 248.0 & 19.2 & 15.7 \\
\hline 1.00 & 156.0 & 92.6 & 248.0 & 77.0 & 15.7 \\
\hline 1.10 & 11.0 & 237.0 & 248.0 & 222.0 & 15.7 \\
\hline
\end{tabular}

\section{Sugestões de atividades}

Algumas perguntas simples podem orientar os estudantes a observar com mais atenção detalhes dos gráficos. Uma observação mais atenta, além de revelar sutilezas a respeito do movimento e ajudar o estudante a incorporar conceitos e relações entre eles, ajuda a desenvolver uma habilidade fundamental na sociedade de informação em que vivemos.

São muitas as perguntas que podem ser feitas e respondidas a partir da observação direta dos gráficos, entre elas:

- Qual o tempo necessário para que a esfera chegue (pela primeira vez) ao ponto mais baixo da sua trajetória?

- Com que velocidade chega neste ponto?

- Qual o tempo necessário para que a esfera chegue ao ponto mais alto de sua trajetória?

- Com que velocidade chega neste ponto?

- Qual a distância percorrida $x$ até o início da subida do looping (que é o ponto mais baixo da trajetória)? E a distância $y$ ? E a distância total $\left(d=\sqrt{x^{2}+y^{2}}\right)$ ?

- Em que pontos da trajetória as velocidades $v_{x c m}$ e $v_{y c m}$ se anulam?

- Em que pontos o módulo da velocidade $\left(|v|_{\mathrm{cm}}\right)$ se anula?

- Em que trechos da trajetória a aceleração é constante? 
- Em que pontos da trajetória a aceleração é nula?

- Em que pontos ou trechos a aceleração é máxima? Qual o valor da aceleração nestes pontos/trechos?

- Escolha três instantes de tempo e verifique se há a conservação da energia mecânica (isto é, se a soma da energia cinética com a energia potencial é de fato a mesma em todos os pontos).

- Escolha três instantes de tempo e determine a razão entre a energia cinética de translação e de rotação. Ela permanece constante ou varia ao longo da trajetória?

A partir dos dados coletados é possível solicitar ao estudante que construa algumas grandezas indiretas:

- Qual a velocidade média da esfera na rampa?

- Qual a velocidade média no looping propriamente dito?

- Qual a velocidade média no circuito?

O procedimento de coleta de dados sugerido acima pode ser feito de maneira sistemática, gerando tabelas e gráficos, como a da Fig. 8, que mostra o tempo que a esfera leva para atingir o ponto mais baixo da trajetória em função da altura de lançamento para esferas de diferentes tamanhos. $\mathrm{O}$ mesmo poderia ser feito com o tempo necessário para que a esfera percorra o looping, por exemplo.

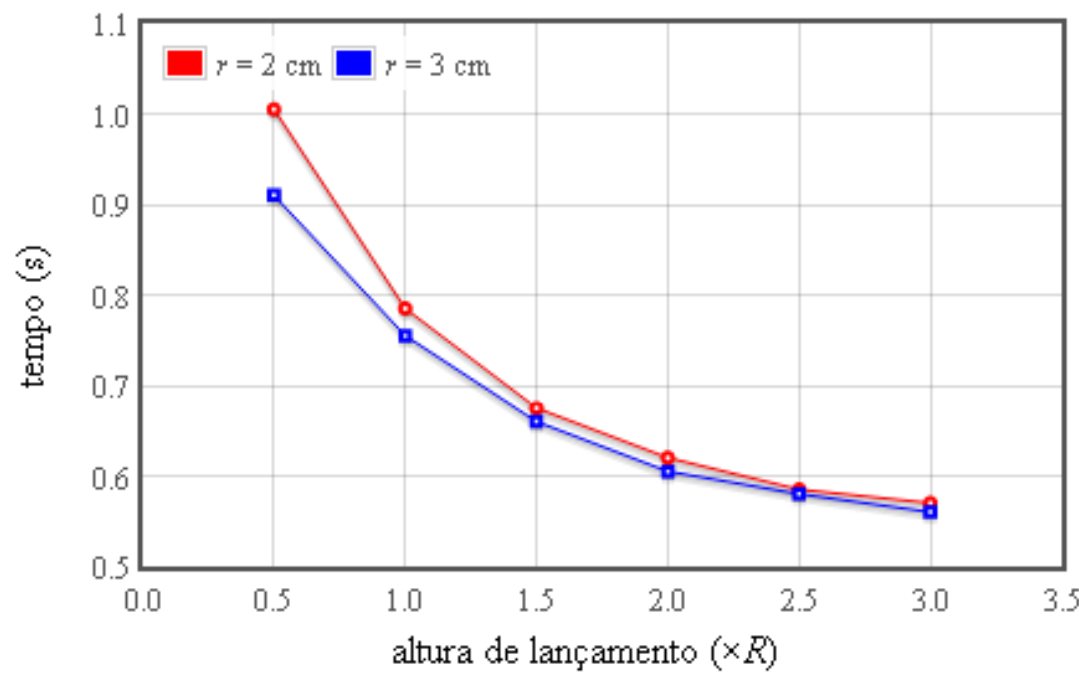

Fig. 8-Gráfico do tempo que a esfera leva para atingir o ponto mais baixo da trajetória, em função da altura de lançamento para esferas de dois tamanhos diferentes.

Os dados disponibilizados nas tabelas permitem a construção de alguns gráficos mais incomuns que podem oferecer outros modos de "enxergar" o movimento. A Fig. 9, por exemplo, 
traz um gráfico das componentes da aceleração em função das respectivas componentes da velocidade enquanto a esfera percorre o looping propriamente dito (o trecho circular da trajetória). São apresentados dados para $H=2,7 R$, limiar de possibilidade de a esfera atravessar o looping.

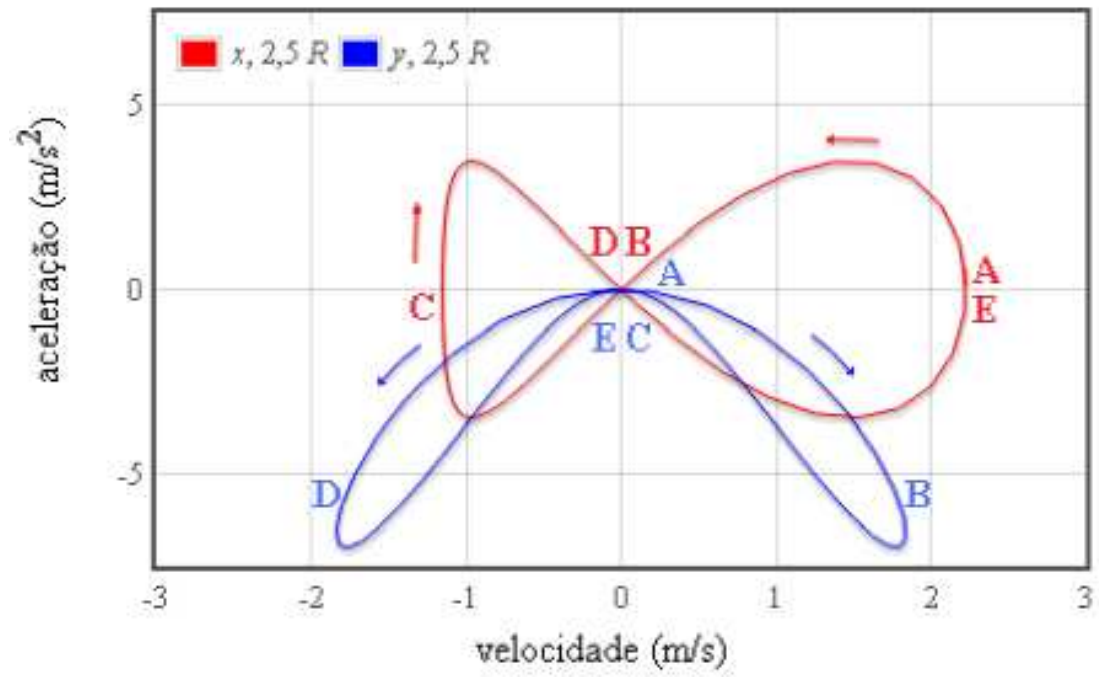

Fig. 9 - Gráficos das componentes da aceleração em função das respectivas componentes da velocidade enquanto a esfera percorre o trecho circular da trajetória para um lançamento a partir de $H=2,7 R$, limiar de possibilidade da esfera atravessar o looping. As marcações correspondem ao ponto mais baixo da trajetória (A), a meio caminho da subida (B), no topo do looping $(C)$, a meio caminho da descida $(D)$ e novamente no ponto mais baixo da trajetória $(E)$.

Um exercício interessante pode ser buscar identificar, nos gráficos, os trechos da trajetória. No início do intervalo considerado, a esfera está "no chão", com sua velocidade horizontal máxima, velocidade vertical nula e as duas componentes da aceleração nulas (A). À medida que sobe o looping, sua velocidade horizontal diminui e a velocidade vertical aumenta. A meio caminho da subida, a velocidade e a aceleração horizontais são nulas (B). A caminho do topo, a velocidade horizontal aumenta para a esquerda, atingindo um máximo (negativo) no topo do looping, enquanto a velocidade vertical vai a zero (C). Na descida, a velocidade horizontal continua negativa, mas diminui em módulo, voltando a ser nula no meio do caminho, onde a velocidade vertical tem um máximo negativo (D). No final do looping a velocidade horizontal é novamente máxima (positiva), e a velocidade vertical e as duas componentes da aceleração se anulam (E).

As Fig. 10 e 11 mostram gráficos um pouco mais simples mas nem por isso menos interessantes para o estudante de Ensino Médio. Diferentemente do exemplo anterior (Fig. 9), em que há a superposição de pontos em apenas um ponto (a origem), nestes dois exemplos é interessante observar que todos os pontos da relação estão sobre um mesmo traço, não importando em que trecho da trajetória a esfera está. 


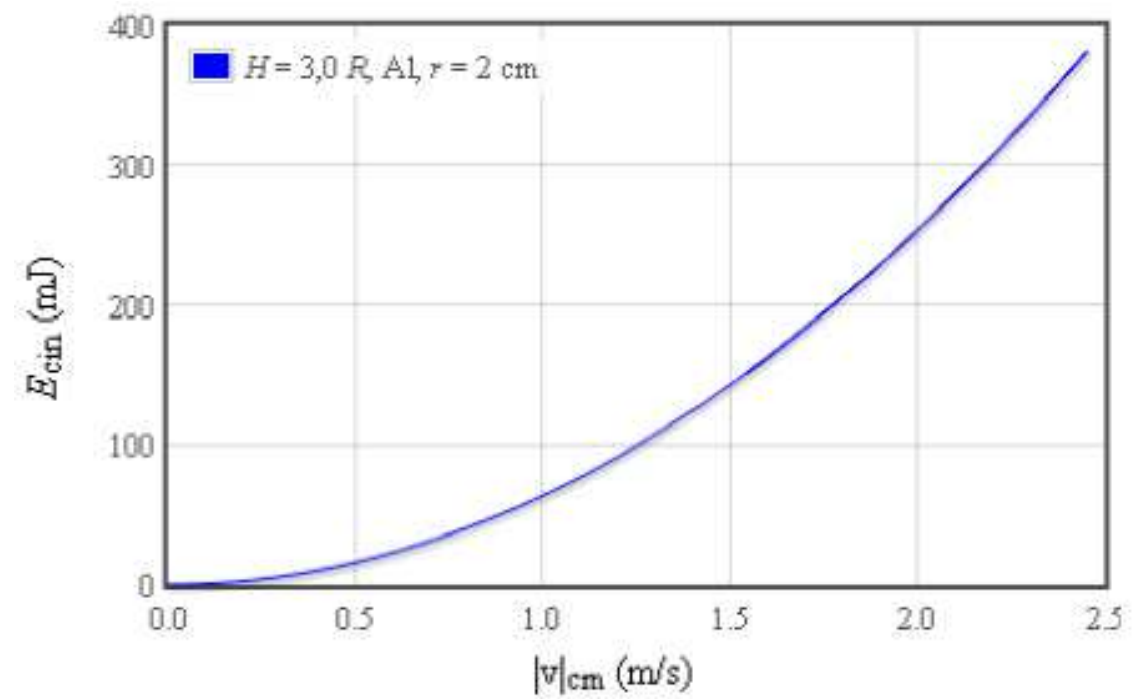

Fig. 10 - Gráfico da energia cinética em função do módulo da velocidade, ao longo de toda a trajetória, para um lançamento a partir da altura máxima permitida pela simulação. Como a energia cinética é sempre proporcional ao quadrado da velocidade, todos os pontos caem sobre a mesma curva $\left.E_{\text {cin }}=\frac{1}{2}\left(1+\frac{2}{5}\right) m v_{c m}\right)$, independentemente do trecho da trajetória em que se encontram.

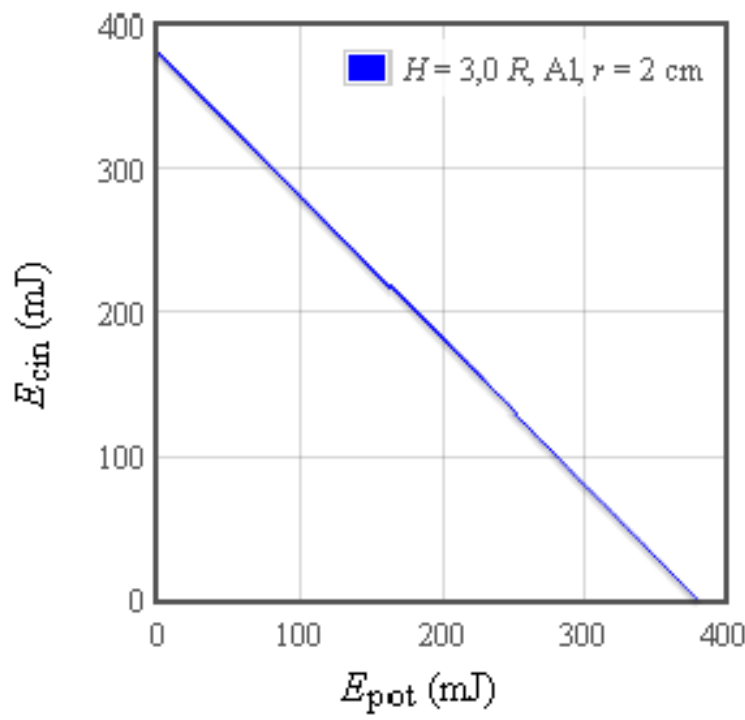

Fig. 11 - Gráfico da energia cinética em função da energia potencial, ao longo de toda a trajetória, para um lançamento a partir da altura máxima permitida pela simulação. Como há uma relação linear entre elas e a energia total do sistema é constante, todos os pontos caem sobre a mesma reta $\left.E_{c i n}=E_{\text {tot }}-E_{\text {pot }}\right)$, independentemente do trecho da trajetória em que se encontram. 
Estes são apenas alguns exemplos dentre muitos outros gráficos que podem ser gerados a partir das tabelas de dados. Estimular os estudantes a fazê-los e interpretá-los é não só uma oportunidade de convívio e fixação dos conceitos físicos subjacentes, mas também uma oportunidade para o desenvolvimento de habilidades de utilização de planilhas de cálculos, por exemplo, para a manipulação de dados e construção de gráficos em geral.

\section{Conclusão}

Neste trabalho exploramos em profundidade o looping, um clássico aparato encontrado em muitos laboratórios didáticos e de demonstrações de fenômenos físicos. Demonstramos como obter as equações da trajetória da esfera no looping utilizando o formalismo lagrangiano, que poucos licenciados em física têm oportunidade de conhecer. Uma vez obtidas as equações, implementamos uma simulação computacional do sistema visando sua utilização como recurso didático para o ensino de física no Ensino Médio ou nas fases iniciais do Ensino Superior e sugerimos algumas estratégias de uso. A simulação pode ser acessada em canzian.fsc.ufsc.br/looping.

A simulação permite que o usuário controle alguns parâmetros do sistema (altura de lançamento, raio e massa da esfera e intervalo de amostragem de dados), apresentando gráficos e tabelas de suas variáveis cinemáticas e dinâmicas (posição, velocidade, aceleração e energia em suas diversas manifestações). As tabelas geradas pela simulação contém uma grande quantidade de dados que podem ser analisados e combinados de várias maneiras, possibilitando a compreensão do fenômeno a partir de diferentes perspectivas, eventualmente auxiliando na incorporação, por parte do aluno, do significado das grandezas físicas exploradas.

\section{Referências}

ARBOR Scientific Loop-the-loop. Ann Arbor: 2015. Disponível em: <http://www.arborsci.com/loop-the-loop>. Acesso em: 29 abr. 2015.

ASAVAPIBHOP, B.; SUWONJANDEE, N. Loop-the-loop: an easy experiment, a challenging explanation. In: INTERNATIONAL CONFERENCE ON PHYSICS EDUCATION (ICPE2009), 2010, Bangkok. p. 249-252. Proceedings...

BOS, B et al. (Eds.) Cascading Style Sheets Level 2 Revision 1 (CSS 2.1) Specification, W3C Recommendation 07 June 2011. Cambridge: W3C, 2011. Disponível em: <http://www.w3.org/TR/CSS21/>. Acesso em: 29 abr. 2015.

CIÊNCIA PRIMA. LBE10 - Looping. Atelier de Experimentos Científicos e Educacionais. São Paulo: 2015. Disponível em: <http://www.cienciaprima.com.br/>. Acesso em: 29 abr. 2015. 
GOLDSTEIN, H. Classical Mechanics. 3. Ed. Reading: Addison-Wesley, 2001. 680 p.

HICKSON, I. et al. (Eds.) HTML5: A vocabulary and associated APIs for HTML and XHTML. W3C Proposed Recommendation 16 September 2014. Cambridge: W3C, 2014. Disponível em: <http://www.w3.org/TR/html5/>. Acesso em: 29 abr. 2015.

KHAN, S. Loop-the-loop question. Mountain View: Khan Academy, 2015. Disponível em: $<$ https://www.khanacademy.org/science/physics/two-dimensional-motion/centripetal-acceleration-tutoria/v/loop-de-loop-question>. Acesso em: 29 abr. 2015.

LAURSEN, O.; SCHNUR, D. FLOT: an attractive JavaScript plotting for jQuery, v. 0.8.3. Alborg: IOLA, 2015. Disponível em: <http://www.flotcharts.org/>. Acesso em: 29 abr. 2015.

MATTHIAS, L. Loop-the-loop. Cornell University Physics Video Demonstration Database. Ithaca: Cornell University, 2015. Disponível em: <http://courses2.cit.cornell.edu/physicsdemos/secondary.php?pfID=43>. Acesso em: 29 abr. 2015.

MUNGAN, C. E.; LIPSCOMBE, T. C. Dropping a particle out of a roller coaster. European Journal of Physics, v. 35, 2014. 045007 (6pp), DOI:10.1088/0143-0807/35/4/045007.

NEELY, A. (Ed.). Loop-the-loop. Cambridge: MIT Technical Service Group, 2015. Disponível em: <https://www.youtube.com/watch?v=dA_UO86MjLY>. Acesso em: 29 abr. 2015.

PRESS, W. H.; TEUKOLSKY, S. A.; VETTERLING, W. T.; FLANNERY, B. P. Numerical Recipes. 3. ed. Cambridge: Cambridge University Press, 2007. p. 907-910. Disponível em: <http://apps.nrbook.com/empanel/index.html>. Acesso em: 29 abr. 2015.

RAGHUNATHAN, R. Loop-the-loop. Albuquerque: University of New Mexico, 2015. Disponível em: <http://physics.unm.edu/pandaweb/demos/questions/predictive3/index.html>. Acesso em: 29 abr. 2015.

SUWONJANDEE, N.; ASAVAPIBHOP, B Loop-the-loop: bringing theory into practice. Physics Education, v. 47, n. 6, p. 751-754, 2012.

SYMON, K. R. Mecânica. Rio de Janeiro: Editora Campus, 1982. p. 389-409.

VARIESCHI, G. U. The projectile inside the loop. Physics Education, v. 41, n. 3, p. 236-239, 2006.

WIRFS-BROCK, A. (Ed.) ECMA-262 ECMAScript Language Specification. 6. ed. June 2015. Geneva: ECMA International, 2015. Disponível em: <http://www.ecma-international.org/publications/files/ECMA-ST/Ecma-262.pdf>. Acesso em: 06 out. 2015. 